\title{
Asynchronous Motor Modeling and Dynamic Co - Simulation Based on ANSYS
}

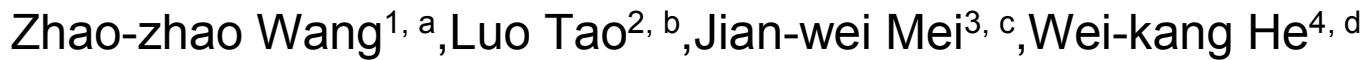 \\ $(1,2,3,4 \mathrm{C}$ ollege of Electrical and Information Engineering, Hubei University of Automotive \\ Technology, Shiyan 442002China)

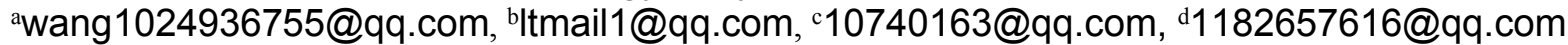

\begin{abstract}
Key words: ANSYS; three-phase asynchronous motor; modeling; simulation
Abstract: Three-phase asynchronous motor simulation model was established based on ANSYS software, the effect factor on the modeling of three-phase asynchronous motor was analyzed in this paper, such as the physical environment to create, geometric model of the stator, rotor geometry model, the asynchronous motor rotor field oriented vector control system model is established with the help of matlab, the collaborative simulation technology were analyzed with the help of ANSYS and matlab. the experimental results show that the experiment platform make abstract concepts more clear and intuitive, meet the requirements of the motor test system.

The design, proofing, validation and modification is the Classic motor design and experimental methods, motor development and experiment cycle is long, cost is high, the magnetic pole shape and tooth distribution of the modern motor is complex and diverse, it make the magnetic circuit complex, at the same time because of the uneven distribution of magnetic flux, the distortion and the local loss of permanent magnet etc, the classic motor design and experimental methods already can't satisfy the need. Aiming at the key problem in the motor design and experiment, the asynchronous motor stator, rotor, and the air gap model will be established using ANSYS software, through the joint simulation between the ANSYS and other software simulation, the magnetic field distribution, the inductance of the motor, torque, etc of the grid division of each node will be accurately calculated, rejection of heat, mechanical properties and electromagnetic properties of the motor will be analyzed.
\end{abstract}

\section{ANSYS SOFT}

ANSYS integration design solutions for motor and control system can solve the rapid design of motor and control system, scheme optimization and precise electromagnetic performance analysis, etc. Users can also add structure, fluid, noise analysis and optimization design software according to need, it will be realized, such as collaborative design in one-way or two-way motor more physical field, high precision motors and control system integration design, multidisciplinary optimization and robust design, etc. ANSYS is also the only software developers that it can be able to provide a complete and advanced motor and control system integrated design platform in the world. It has functions for motor mechanical and electrical system designing and analyzing, such as:

(1) rotary motor design expert RMxprt

(2) The electromagnetic field finite element analysis software Maxwell

(3) Circuit and system simulation software Simplorer

(4) High performance computing

(5) A key finite element

(6) adaptive meshing 


\section{modeling Three-phase asynchronous motor experiment platform}

Modeling experiment platform

Three-phase asynchronous motor experiment system include: asynchronous motor ontology modeling, control system modeling, collaborative simulation technology and experiment results analysis and processing, the modeling process are shown in figure 1.

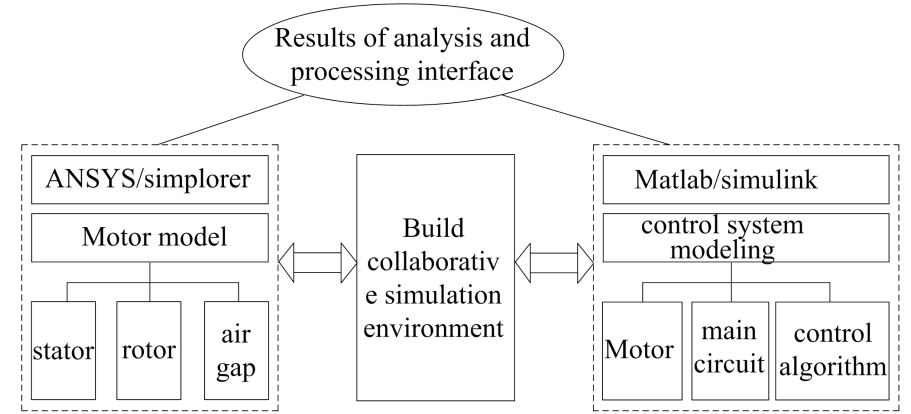

Fig. 1 the experimental system modeling process

three-phase asynchronous motor modeling method

The essence of the asynchronous motor model based on ANSYS is making motor run in the computer, the basic steps: pretreatment (modeling), the meshing, load solving and post-processing.

Modeling by ANSYS for asynchronous motor has two kinds of methods, one is to use the GUI control flow, modeling according to the shortcut menu of the graphical interface, Second is to mode by the command flow.

In the modeling of the motor, the first to establish the component part of asynchronous motor, such as stator core, stator winding, rotor, the rotor core, air gap, etc., the second to divide the grid and define unit attribute of each part, ensure the integrity of the motor model. Three-phase asynchronous motor modeling process is shown in figure 2 .

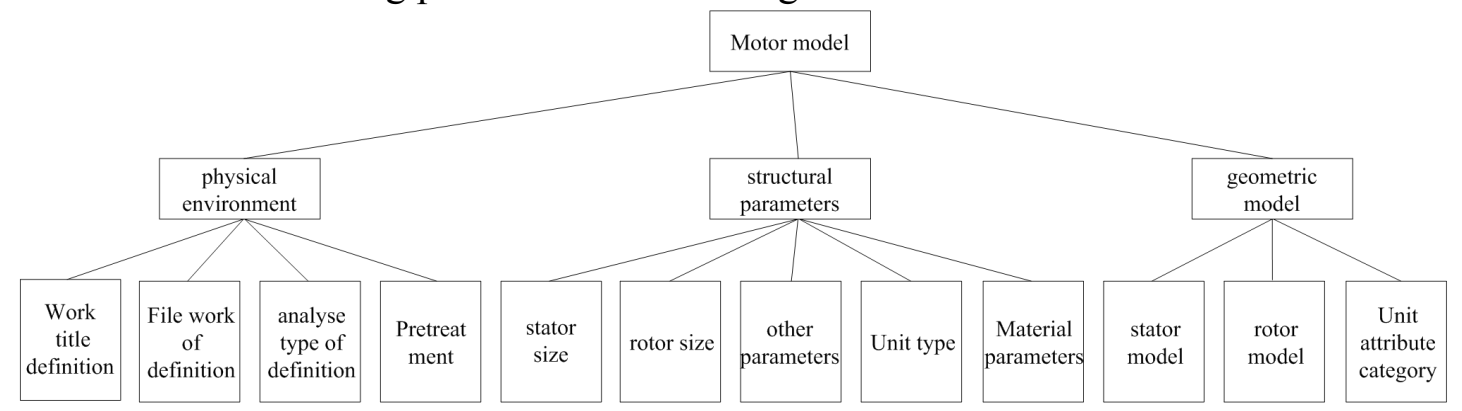

Fig. 2 Asynchronous motor modeling process

\section{Asynchronous motor modeling steps}

the creation of physical environment

Physical environment creation is the foundation of modeling the motor, including: definition of job title, file name and the type of analysis, the top processing, parameter Settings of asynchronous motor stator and rotor basic, definition of parameters and unit type and the definition of the component characteristics.

(1) The stator dimensions

the stator structure size is defined Using the command line statements, including the outer and inner diameter of the stator core, the number of stator slot, the stator slot height, the stator slot width, the dimensions of the stator slot and the stator slot width, such as command line statements of defining core diameter is: outer_stator $=1400 / 1000$, the stator slot number: nslot_stator $=72$, the 
stator slot width: ss_w1 $=20 / 1000$.

(2) The rotor dimensions

Using the command line statements for defining counter rotating substructure size, including the rotor core diameter, inner diameter, the number of rotor slot, the rotor slot height, the rotor slot width (up, down), such as command line statements for rotor core inner diameter: inner_rotor $=$ 500/1000, the rotor slot height: $\mathrm{sr}$. $\mathrm{h}=51.5 / 1000$.

(3) Other parameter Settings

Using command line statements for defining other important parameters, including the phase number, core length, parallel branch route number, wire width, height of conductor, and the number of round wire and pitch, coil turns number, winding filling coefficient, trapezoidal conductor height, trapezoidal conductor bottom width of up and down, cross-sectional area of trapezoidal conductor, conductor axial length,such as the number of command line statements define phase: $\mathrm{m} \_$phase $=3$, logarithmic: $n \_$pole $=4$, wire width: ss_clw $=16 / 1000$, and parallel branch route number: ss_clh $=$ 4/1000, trapezoidal conductor up bottom width: ww2 = sr_w2, conductor axial length: conducting bar leng2 = leff.

(4) definition of unit type

Using the command line statements for unit type definition, mainly including the definition of four degrees of freedom, such as AZ freedom - for air and insulation area: et, 1,53,0, AZ freedom used for rotor slot conducting bar: et and 3,53,1, AZ freedom - for core areas: et, 4,53,0.

(5) the definition of material parameters

Using the command line statements for defining material parameters, including air magnetic permeability of air and the rotor conductor, resistivity of the stator conductor and the rotor conductor, core materials, such as air Magnetic permeability, mp, murx, 1, 1, the stator conductor permeability: mp, murx, 2, 1, the stator conductor resistance rate: mp, RSVX, 2,0.0434e-6. Create the stator geometric model

After the completion of seting up the physical environment and structure parameters, the geometric model of the stator will created, the following steps is:

(1) Create a stator core area,as shown in figure 3
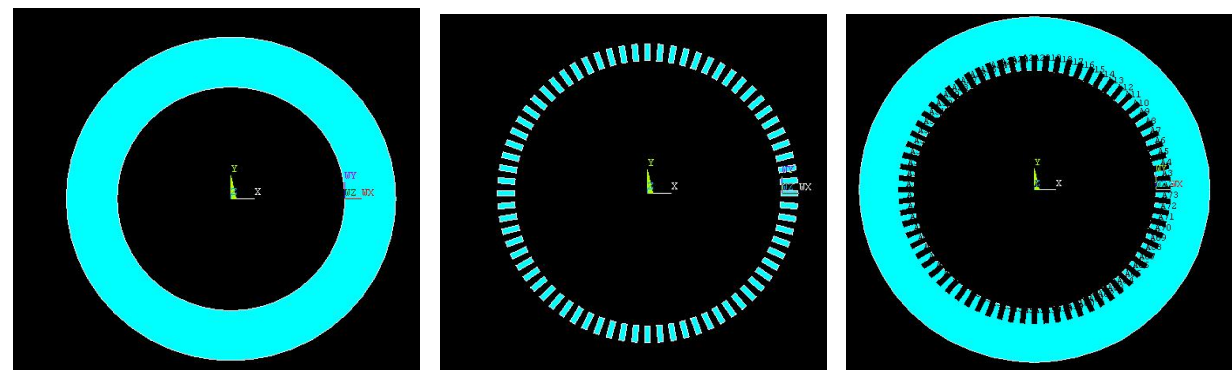

Fig. 3 segmented regions of Concentric circles Fig. 4concentric circles rectangle Fig. 5 Stator core

(2) Create 72 stator slot

By calling csys command the polar coordinates will be getted, a total of 360 degrees, 4.8 degrees between each stator slot, as shown in figure 4 . 


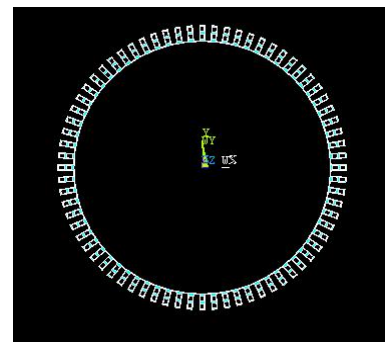

Fig. 6 the air gap

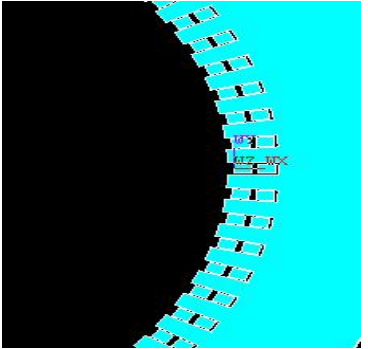

Fig. 7 the stator model

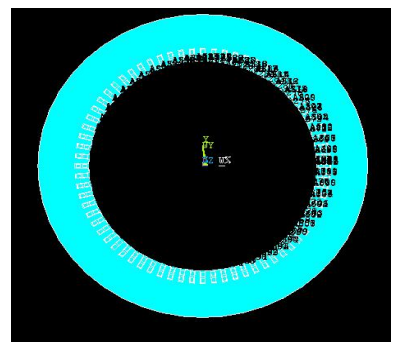

Fig. 8 the rotor model

(3) Create stator core

The complete stator core will be produced by subtracting the components of the core and the stator slot by subtracting, as shown in figure 5 .

(4) Create the air gap of the stator

The concentric zone will be formed by subtracting the inner radius diameter of stator core and the radius of the air gap, the air gap will be getted by subtracting the above areas and the stator iron core,gap model is shown in figure 6.

Bai Kong in Air-gap figure is the coil, the coil is divided into two layers, as shown in figure 7. The rotor geometry model creation

The steps to create the geometric model of the rotor is similar to stator geometric model steps, repeat the above steps, the modeling on the command line statement shall be redefined in accordance with the size and parameters of the rotor core and winding, the rotor iron core model as shown in figure 8.

Unit attribute category

When various parts of the asynchronous motor components are modeling is completed, the next work is to divide and define various parts of the component unit attribute category, its command statements are shown in table 1.

table 1 Unit attribute definition command

\begin{tabular}{llll}
\hline $\begin{array}{c}\text { command } \\
\text { statements one }\end{array}$ & \multicolumn{1}{c}{$\begin{array}{c}\text { command statements } \\
\text { two }\end{array}$} & \multicolumn{1}{c}{$\begin{array}{c}\text { command statements } \\
\text { three }\end{array}$} & \multicolumn{1}{c}{$\begin{array}{c}\text { command } \\
\text { statements four }\end{array}$} \\
\hline cmsel,s,air1 & lesize,all,length1 & mshape,1,2d & mshape,1,2d \\
cmsel,a,air2 & mshape,1,2d & SMRTSIZE,2 & SMRTSIZE,2 \\
aatt,1,1 & SMRTSIZE,2 & amesh,all & amesh,all \\
cmsel,s,winding1 & amesh,all & cmsel,s,iron2 & cmsel,s,iron1 \\
aatt,2,2,2 & cmsel,s,air1 & lsla,s & 1sla,s \\
cmsel,s,winding2 & lsla,s & lsel,r,loc,x,inner_rotor/2 & lesize,all,20 \\
aatt,3,3,3 & lesize,all,length1 & lesize,all,,,10 & mshape,1,2d \\
cmsel,s,iron1 & mshape,1,2d & cmsel,s,iron2 & SMRTSIZE,4 \\
cmsel,a,iron2 & SMRTSIZE,2 & lsla,s & amesh,all \\
aatt,4,,4 & amesh,all & lesize,all,length1 & allsel \\
allsel & cmsel,s,winding2 & mshape,1,2d & \\
cmsel,s,air2 & 1sla,s & SMRTSIZE,4 & \\
lsla,s & lsel,r,length,,sr_h-sr_hh1 & amesh,all & \\
length1=airgap/2 & lesize,all,length1*2 & cmsel,s,winding1 & \\
\hline After the command & executing,thesimutation & \\
\hline
\end{tabular}

After the command executing, the simulation model and results of induction motor can be obtained, as shown in figure 9, 10 . 


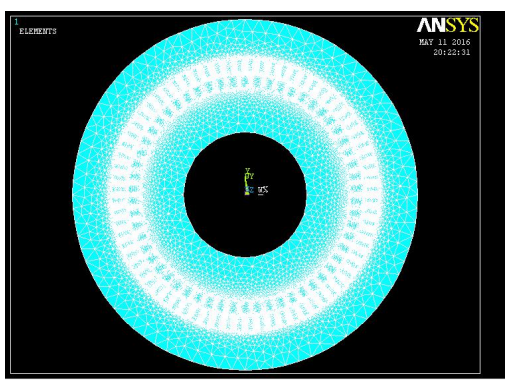

Fig. 9 Induction motor model

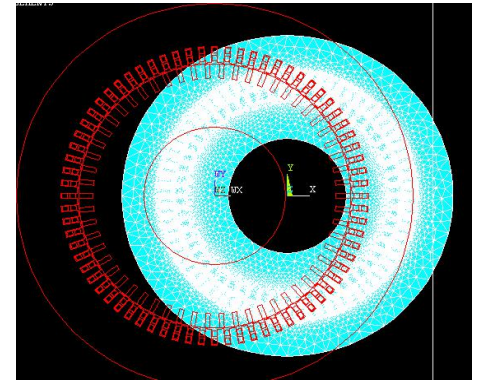

Fig. 10 the simulation results

\section{Collaborative simulation technology}

simulation model of three-phase asynchronous motor control system is Established in Matlab/Simulink. Using ANSYS and Matlab, Maxwell, RMxpt software interface, it will realize the coordination simulation between different software and is helpful to transfer between model and work together between the groups, it not only can avoid a repeat of the modeling work, but also can make full use of the software design advantages expand software design capabilities, to meet the designers requirements in various fields, multi-level and many organization.

Induction motor vector control model is established in Matlab, before the joint simulation, AnsoftLinkDialog, AnsoftSFunction.dll and AnsoftSFunction will be loaded into the Matlab scanning path in Simplorer file.

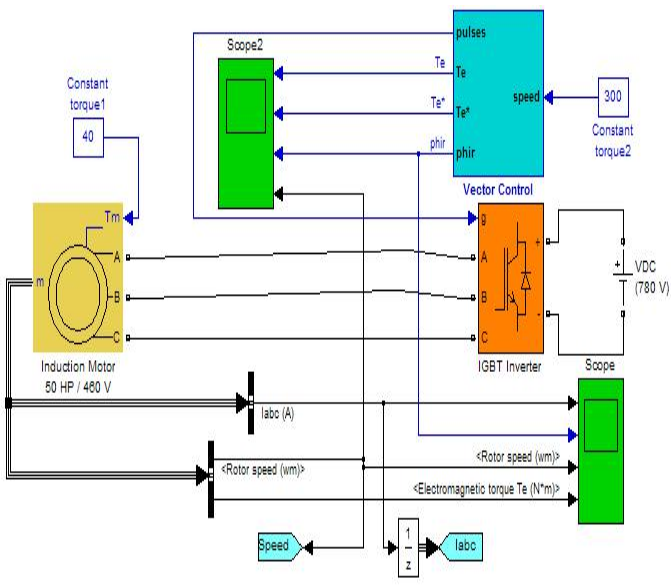

Fig. 11 Vector control model

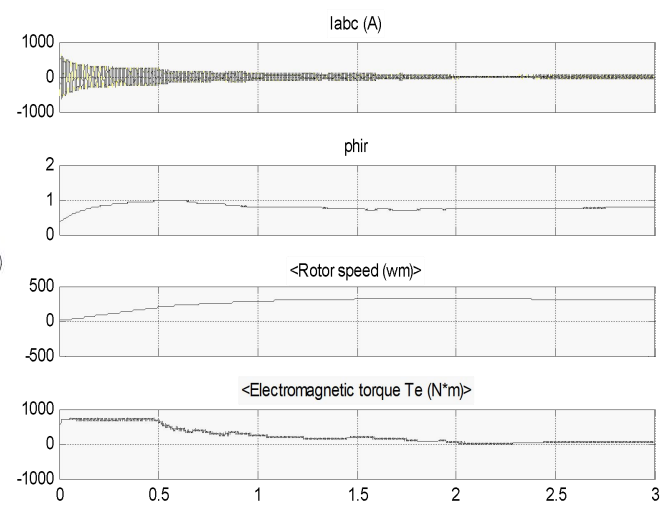

Fig. 12 The simulation waveform

\section{Conclusion}

The stator, rotor and crack model of three-phase asynchronous motor is established by use of ANSYS software, using the ANSYS software tools the electromagnetic field distribution are simulated, by combining with other motor control application software, the three-phase asynchronous motor mechanical and electrical control system can be collaborative simulation, the establishment of the virtual experiment platform can not only promote the deep understanding of the motor and its structure, but also lay a solid foundation for the motor design and performance analysis.

\section{Acknowledgements}

This work won the key discipline project in Hubei Province ---Research on Key Fundamental 
Problems in Parameter Identification and Decoupling Control of Brushless Doubly - Fed Machine(D20171802)funding.

\section{References}

[1] ZHAO Lu. Co-simulation based on Simplorer field-circuit coupled physics field[M]. beijing: China Water\&Power Press, 2014.3.

[2] Hu Renxi, Sun Mingli.ANSYS13.0 electromagnetic finite element analysis from entry to the master[M]. beijing: China Machine Press, 2012.1

[3] Xia Zhengze, Liu Huijuan. Based on the field-circuit coupling method of asynchronous traction motor electromagnetic field analysis[J]. Micromotors, 2009.3(24).

[4] Yan Zhaowen.ANSYS10.0 electromagnetic analysis technology and engineering example explanation[M]. beijing: China Water\&Power Press, 2006.11.

[5] Liu Xuemei, Ni Zehao, Li Aiping. Based on large induction motor rotor system dynamic characteristic analysis of ANSYS[J].Chinese Journal of Construction Machinery,2013,06:480-484.

[6] Wang Xinwei, Liu Wensheng, Wang Yi. ANSYS software in the application of the bearingless induction motor magnetic field analysis[J].Electrical Machinery Technology,2008,02:13-16. 